\title{
Philosophiques
}

\section{La Forme méditative de la métaphysique cartésienne}

\section{Laurence Devillairs}

Volume 28, numéro 2, automne 2001

URI : https://id.erudit.org/iderudit/005666ar

DOI : https://doi.org/10.7202/005666ar

Aller au sommaire du numéro

Éditeur(s)

Société de philosophie du Québec

ISSN

0316-2923 (imprimé)

1492-1391 (numérique)

Découvrir la revue

Citer cet article

Devillairs, L. (2001). La Forme méditative de la métaphysique cartésienne. Philosophiques, 28(2), 281-301. https://doi.org/10.7202/005666ar

\section{Résumé de l'article}

Descartes choisit de donner à sa première publication métaphysique, la forme et le titre de " méditations ». Cette dénomination est un sujet d'études traditionnel du commentaire cartésien qui l'associe généralement à un exercice religieux, issu de la spiritualité jésuite. Nous souhaiterions renouveler cette interprétation et montrer que la forme méditative de la métaphysique n'est ni une rhétorique ni une pratique religieuse mais qu'elle représente une méthode proprement philosophique. De plus, ce n'est pas aux exercices d'Ignace de Loyola que semble se référer la méditation cartésienne mais bien plutôt à la pensée d'Augustin.
Ce document est protégé par la loi sur le droit d'auteur. L'utilisation des services d'Érudit (y compris la reproduction) est assujettie à sa politique d'utilisation que vous pouvez consulter en ligne.

https://apropos.erudit.org/fr/usagers/politique-dutilisation/ 


\title{
La Forme méditative de la métaphysique cartésienne
}

\author{
LAURENCE DEVILLAIRS \\ École Normale Supérieure \\ Ldevill@aol.com
}

\begin{abstract}
RÉSUMÉ. - Descartes choisit de donner à sa première publication métaphysique, la forme et le titre de « méditations ». Cette dénomination est un sujet d'études traditionnel du commentaire cartésien qui l'associe généralement à un exercice religieux, issu de la spiritualité jésuite. Nous souhaiterions renouveler cette interprétation et montrer que la forme méditative de la métaphysique n'est ni une rhétorique ni une pratique religieuse mais qu'elle représente une méthode proprement philosophique. De plus, ce n'est pas aux exercices d'Ignace de Loyola que semble se référer la méditation cartésienne mais bien plutôt à la pensée d'Augustin.
\end{abstract}

\begin{abstract}
Descartes chose to give to his first metaphysical publication, the form and the title of meditations. This denomination is a traditional topic of Cartesian commentary, which generally relates it to a religious exercise, derived from Jesuitical spirituality. We wish for changing this interpretation by asserting that the meditative form of Metaphysics is not a question of rhetoric neither of religious practice but it represents a purely philosophical method. Moreover, it is not to Ignace de Loyola's exercises that Cartesian meditation seems to refer but rather to Augustine's thought.
\end{abstract}

La forme méditative a donné son nom à l'ouvrage de philosophie première de Descartes, associant dans un même acte, recherche métaphysique et attitude méditative. Cette association sera cependant rompue ultérieurement pour être remplacée par un exposé dogmatique, celui des Principia : la forme méditative semble alors frappée de contingence - la philosophie première pouvant tout aussi bien être présentée sous une forme scolaire ${ }^{1}$. Aussi, à traiter de la forme méditative de la métaphysique cartésienne, semblons-nous condamnés à choisir entre deux types d'interprétation : l'une pauvre qui considère la méditation comme simple titre pour désigner l'exercice métaphysique et l'assimiler ainsi à un problème rhétorique. L'autre qui voit dans la méditation cartésienne l'équivalent d'un exercice religieux ayant son origine dans la spiritualité d'Ignace de Loyola.

1. Dès septembre 1640, avant l'envoi du manuscrit des Meditationes, Descartes confie à Mersenne son projet de rédiger un exposé complet en forme de cours de sa philosophie, à la manière des manuels jésuites et avec pour but de s'y substituer, voir Lettre à Mersenne, 30 septembre 1640. Nous citerons Descartes à partir de l'édition des Euvres de Descartes, publiées par C. Adam et P. Tannery, Paris, Vrin, 1996, abrégé [AT], suivi du tome en chiffres romains [III] et de la page en chiffres arabes [185]. 
Nous souhaiterions pour notre part proposer une autre interprétation - qui accorde à la fois suffisamment à la forme méditative pour ne pas la réduire à une question stylistique; et qui en précise le sens non pas spirituel ou religieux mais proprement métaphysique. Nous développerons l'hypothèse selon laquelle la forme méditative de la métaphysique cartésienne correspond à une opération intellectuelle spécifique à la connaissance de Dieu et exprime ainsi la volonté cartésienne de parvenir à une connaissance intuitive de la nature divine - et ce jusque dans l'exposé des Principes.

Toutefois, en adoptant cette forme et en lui conférant sa légitimité philosophique, Descartes ne se trouve pas sans prédécesseur, mais retrouve le mode méditatif propre à la pensée d'Augustin : de fait, dans la présentation qu'il donne des preuves de l'existence de Dieu - notamment dans le De Libero arbitrio - l'évêque d'Hippone mêle démonstration et méditation. C'est sur le fondement de cette épistémologie que nous semble s'élaborer également le système cartésien des preuves de l'existence de Dieu.

\section{La Méditation comme réforme de la philosophie et comme méthode}

La connaissance de la nature divine et l'adoption de la forme méditative pour exposer la philosophie première est indissociable du tournant de la philosophie cartésienne, opéré au cours de l'année 1628-1629. Ce tournant se marque par l'attribution aux vérités métaphysiques d'une évidence supérieure à celle des vérités mathématiques ${ }^{2}$. Aussi, est-il possible de séparer nettement un avant d'un après dans l'élaboration de la métaphysique : la Règle II pose l'évidence mathématique comme norme pour toute connaissance qui se voudrait aussi science ${ }^{3}$. L'arithmétique et la géométrie seules exhibent le chemin qui conduit à la vérité et toute connaissance qui emprunte ce chemin aura pour fin d'accéder à une certitude équivalente "aux démonstrations de l'arithmétique et de la géométrie " ${ }^{4}$. Mais que l'équivalence corresponde à un maximum, c'est-à-dire au degré ultime de certitude auquel puisse atteindre toute connaissance autre que l'arithmétique et la géométrie, repose sur la supériorité non de droit mais de fait de ces deux sciences. En effet, aucune inquiétude métaphysique ne vient mettre en cause leurs vérités qui paraissent indubitata ${ }^{5}$; aucun questionnement sur la capacité de l'entendement à

2. «( (...) au moins pensé-je avoir trouvé comment on peut démontrer les vérités métaphysiques, d'une façon qui est plus évidente que les démonstrations de géométrie ", A Mersenne, 15 avril 1630, AT I, 144.

3. "Toute science est une connaissance certaine et évidente ", Regulce, AT X, 362.

4. AT X, 366.

5. AT X, 362. Pour transformer cette certitude de fait (indubitata) en indubitabilité de droit, il convient de mettre en question d'abord la possibilité d'une science qui ne serait pas fondée sur la connaissance de Dieu, ensuite la capacité de l'entendement qui devra alors faire 
atteindre la vérité n'est réellement formulé. Or seul un tel questionnement peut provoquer l'unique raison métaphysique de douter ${ }^{6}$. C'est sur ce point, à l'inverse, que s'inaugureront les Méditations au travers des hypothèses athées et du Dieu tout-puissant ${ }^{7}$.

Les Méditations présenteront la preuve ontologique comme au moins aussi certaine ${ }^{8}$ que les démonstrations mathématiques et donneront ainsi raison à l'assurance qui animait plusieurs lettres de $1630^{9}$. La Cinquième Méditation, par la comparaison entre les essences mathématiques et l'essence divine - laquelle seule implique l'existence nécessaire - , décide de façon définitive de l'ordo rationum : toutes les sciences, même la mathématique, supposent comme leurs fondements la connaissance de Dieu et de l'âme. La métaphysique, mieux encore que les mathématiques, est donc en mesure de déterminer une "voie de déduction rationnelle ». C'est donc désormais pour qualifier cette connaissance que Descartes use d'un lexique auparavant réservé à l'arithmétique et à la géométrie ${ }^{10}$.

Cette exigence de réforme exprimée au travers de l'adoption de la forme méditative constitue-t-elle un récit de conversion ? Est-il pertinent d'associer la méditation cartésienne à la méditation religieuse ${ }^{11}$ ? Pour pré-

l'épreuve de la détermination de son origine. Telles seront les « expériences métaphysiques » de la Première Méditation, AT IX, 16-17, des articles 5 et 30 des Principes (où les évidences mathématiques ont été soumises au doute hyperbolique, summa dubitatio).

6. « Nous douterons aussi de toutes les autres choses qui nous ont semblé autrefois très certaines, même des démonstrations de mathématique et de ses principes, encore que d'eux-mêmes ils soient assez manifestes, parce qu'il y a des hommes qui se sont mépris en raisonnant sur de telles matières ; mais principalement parce que nous avons ouï dire que Dieu, qui nous a créés, peut faire tout ce qui lui plait, et que nous ne savons pas encore s'il a voulu nous faire tels que nous soyons toujours trompés, même aux choses que nous pensons mieux connaître ", Principes I, article 5 . L'ignorance du vrai Dieu c'est-à-dire de l'auteur de notre être élève le doute à son paroxysme.

7. « (...) il y a un Dieu qui peut tout, et par qui j’ai été créé et produit tel que je suis ", AT IX, 16 ; " Toutefois, de quelque façon qu'ils supposent que je sois parvenu à l'état et à l'être que je possède, soit qu'ils l'attribuent à quelque destin ou fatalité, soit qu'ils le réfèrent au hasard, soit qu'ils veuillent que ce soit par une continuelle suite et liaison des choses (...) ", ibid.

8. AT IX, 52 ; même expression dans le Discours, IV, AT VI, 36.

9. Outre la lettre à Mersenne du 15 avril 1630, il faut signaler celle qui est adressée au même correspondant du 25 novembre 1630, AT I, 181-182 ainsi que celle à *** de mars 1637 ( ?), AT I, 353. Sur le rapport entre la preuve de la Cinquième Méditation et le commencement de la métaphysique, voir Rodis-Lewis, Geneviève, Le Développement de la pensée de Descartes, Paris, Vrin, 1997, p. 145.

10. Outre le lexique de la certitude et de l'indubitabilité, celui de la facilité sert à décrire l'arithmétique et la géométrie qui «sont ainsi les plus faciles et les plus claires » de toutes les disciplines, AT X, 365. C'est ensuite la connaissance des attributs de Dieu qui est ainsi qualifiée, Réponses aux Premières Objections, AT IX, 94 ; Epître, AT IX, 5 ; Cinquième Méditation, AT IX, 55.

11. "La méditation religieuse révèle de manière caractéristique une personne à la recherche du salut qui, au commencement, se trouve dans l'obscurité du péché, et qui, à travers une conversion est conduite à l'illumination spirituelle (...) C'est à cette sorte de travail qu'appartiennent, d'un point de vue général, les Méditations ", Frankfurt, Harry G., Démons, rêveurs et fous. La défense de la raison dans les Méditations de Descartes, Paris, PUF, 1989, p. 11. 
ciser cette idée d'une conversion dans le récit cartésien, nous prendrons appui sur l'hypothèse du malin génie. Cet artifice méthodologique nous semble exemplaire de la rhétorique de la conversion : il révèle le caractère volontaire du narrateur cartésien ${ }^{12}$ qui, jusque dans l'expérience délétère du doute, éprouve la fermeté de sa décision (douter de tout pour fonder la science). Il donne aussi la preuve qu'il ne peut y avoir de « réforme » de la connaissance qu'en transformant cette décision volontaire en habitude acquise. En effet, pour se défaire de "la sollicitation humble, insinuante et tenace du vraisemblable ${ }^{13}$ et maintenir le doute, il faut contrecarrer la confiance naturelle que nous portons aux opinions vraisemblables ${ }^{14}$ par une défiance artificielle, remplacer une coutume ${ }^{15}$ par une autre ${ }^{16}$. L'acte de la volonté est cette transition entre une coutume et l'adoption d'une attitude qui deviendra une coutume contraire à la précédente. L'habitude métaphysique consiste ainsi, par la méditation, à éloigner l'esprit des sens, «afin que l'habitude de confondre les choses intellectuelles avec les corporelles, qui s'est enracinée en nous pendant tout le cours de notre vie, puisse être effacée par une habitude contraire de les distinguer ${ }^{17}$. Il n'empêche, cependant, que le changement doit être radical, la confiance naturelle supplantée par une défiance méthodique. Toutefois, dans cette substitution d'une habitude à une autre, la passivité a fait place à la maîtrise ${ }^{18}$.

La réforme est donc tout à la fois redressement et habitude. Il n'est donc pas surprenant de retrouver, dans le texte tardif du Traité des Passions, la description de ce même processus d' " accoutumance ", appliqué cette fois à une réforme non de l'entendement mais de la volonté elle-même :

(...) encore que chaque mouvement de la glande semble avoir été joint par la nature à chacune de nos pensées dès le commencement de notre vie, on les peut toutefois joindre à d'autres par habitude (...). Car, puisqu'on peut, avec un peu

12. «Quapropter, ut opinor, non male agam, si, voluntate plane in contrarium versa (...) », AT VII, 22.

13. Gouhier, Henri, Essais sur Descartes, Paris, Vrin, 1937, p. 152-153 sq.

14. «Car ces anciennes et ordinaires opinions me reviennent encore souvent en la pensée, le long et familier usage qu'elles ont eu avec moi leur donnant le droit d'occuper mon esprit contre mon gré, et de se rendre maîtresses de ma créance ", AT IX, 17.

15. «Et je ne me désaccoutumerai jamais d'y acquiescer, et de prendre confiance en elles, tant que je les considèrerai telles qu'elles sont en effet, c'est à savoir en quelque façon douteuses, comme je viens de le montrer et toutefois fort probables, en sorte que l'on a beaucoup plus de raison de les croire que de les nier ", ibid.

16. "C'est pourquoi je pense que j'en userai plus prudemment, si, prenant un parti contraire, j'emploie tous mes soins à me tromper moi-même, feignant que toutes ces pensées sont fausses et imaginaires ", ibid.

17. Réponses aux Secondes Objections, AT IX, 104.

18. "Jusques à ce qu'ayant tellement balancé mes préjugés, qu'ils ne puissent faire pencher mon avis plus d'un côté que d'un autre, mon jugement ne soit plus désormais maîtrisé par des mauvais usages et détourné du droit chemin qui le peut conduire à la connaissance de la vérité », AT IX, 17. 
d'industrie, changer les mouvements du cerveau dans les animaux dépourvus de raison, il est évident qu'on le peut encore mieux dans les hommes, et que ceux même qui ont les plus faibles âmes pourraient acquérir un empire très absolu sur toutes leurs passions, si on employait assez d'industrie à les dresser et à les conduire ${ }^{19}$.

La réforme de la philosophie, qui doit conférer aux preuves métaphysiques une évidence supérieure aux démonstrations géométriques ${ }^{20}$, fonctionne de façon analogue : elle remplace une habitude ancienne par une habitude acquise. Par voie de conséquence, la méditation exprime davantage une "réforme de l'entendement " qu'un exercice religieux.

En effet, la philosophie première ne peut accomplir sa tâche, qui est de démontrer l'existence de Dieu ${ }^{21}$, sans combattre une habitude ancienne qui soumet l'entendement au " commerce des sens ». Ce sera donc par l'adoption d'une habitude contraire, qui consiste à détacher l'esprit de ce " commerce des sens », que les preuves cartésiennes de l'existence de Dieu se développeront et manifesteront leur évidence absolue et leur certitude ${ }^{22}$. C'est parce que la première partie de la science - cette première partie de la science consiste à détruire généralement toutes mes anciennes opinions et à préparer l'esprit, par la méditation et une attention soutenue, à l'entreprise d'établissement des fondements de la science - est soutenue tout entière par cette résolution inaugurale, qu'elle ne doit pas s'abolir une fois le chemin accompli. Les Principes qui présentent pourtant la science cartésienne de façon dogmatique ne gommeront pas comme des scories les étapes de cette première partie de la science. Parce que l'itinéraire n'est pas dissociable de la science, il exige sa réitération par le lecteur : les Principes comme les Méditations supposent au contraire un lecteur à enseigner ${ }^{23}$.

19. Passions de l'âme, Première Partie, article 50.

20. « (...) encore que j’estime que celles [les démonstrations] dont je me sers ici, égalent, voire surpassent en certitude et évidence les démonstrations de géométrie ", Epître, AT IX, 6-7.

21. "J'ai toujours estimé que ces deux questions, de Dieu et de l'âme, étaient les principales de celles qui doivent plutôt être démontrées par les raisons de la philosophie que de la théologie ", ibid., p. 4.

22. «(...) j’appréhende néanmoins qu'elles [les démonstrations métaphysiques] ne puissent pas être assez suffisamment entendues de plusieurs, tant parce qu'elles sont aussi un peu longues, et dépendantes les unes des autres, que principalement parce qu'elles demandent un esprit entièrement libre de tous préjugés et qui se puisse aisément détacher du commerce des sens ", ibid., p. 7, souligné par nous.

23. "Je ne conseillerai jamais à personne de le lire [mon livre] sinon à ceux qui voudront avec moi méditer sérieusement », Préface, trad. de AT VII, 9-10. 


\section{Abducere mentem a sensibus}

En tant que mode d'accès privilégié à Dieu, la méditation constitue une méthode : "Or, qu'il y ait en nous l'idée d'un être souverainement puissant et parfait, et aussi que la réalité objective de cette idée ne se trouve point en nous, ni formellement ni éminemment, cela deviendra manifeste à ceux qui y penseront sérieusement, et qui voudront avec moi prendre la peine d'y méditer ${ }^{24}$. Dans une lettre postérieure à la publication du Discours, Descartes explique de façon nette ce que signifie cette méthode qu'il nomme ad abducendam mentem a sensibus et par laquelle il " pense prouver qu'il n'y a rien au monde qui soit de soi plus évident et plus certain que l'existence de Dieu et de l'âme humaine ». Il souligne les principales étapes de cette méthode : premièrement, il faut « expliquer bien au long les plus fortes raisons des sceptiques, pour faire voir qu'il n'y a aucune chose matérielle de l'existence de laquelle on soit assuré "; deuxièmement, il s'agit " par même moyen [d'] accoutumer le lecteur à détacher sa pensée des choses sensibles ${ }^{25}$. L'aboutissement de ces étapes initiales est l'affirmation du Cogito : « (...) puis montrer que celui qui doute ainsi de tout ce qui est matériel, ne peut aucunement pour cela douter de sa propre existence; d'où il suit que celui-là, c'est-à-dire l'âme, est un être, ou une substance qui n'est point du tout corporelle, et que sa nature n'est que de penser, et aussi qu'elle est la première chose qu'on puisse connaître certainement ». Enfin, si cette méditation est suffisamment développée, " (...) on acquiert peu à peu une connaissance très claire, et si j'ose ainsi parler intuitive, de la nature intellectuelle en général, l'idée de laquelle, étant considérée sans limitation, est celle qui nous représente Dieu ». Parcourir ces trois étapes est la condition nécessaire et suffisante pour parvenir à la connaissance de la nature divine : « (...) il n'est pas possible de bien entendre ce que j'ai dit après de l'existence de Dieu, si ce n'est qu'on commence par là ${ }^{26}$.

La connaissance de Dieu se fait en se détournant, à travers l'épreuve du doute, de la familiarité qui nous lie aux choses matérielles et au monde sensible. En d'autres termes, la connaissance de Dieu est proportionnelle à la capacité de notre entendement à se détacher des sens : « (...) car il n'est pas possible de bien connaître la certitude et l'évidence des raisons qui prouvent l'existence de Dieu selon ma façon, qu'en se souvenant distinctement de celles qui nous font remarquer de l'incertitude en toutes les connaissances que nous avons des choses matérielles ${ }^{27}$. C'est pourquoi la détermination des attributs divins ne peut, en aucun cas, dépendre de considérations cosmologiques.

24. AT IX, 107.

25. A Vatier, 22 février 1638, AT I, 560.

26. Toutes les citations renvoient à la lettre à Silhon ( ?), mars 1637 ( ?), AT I, 353.

27. A Vatier, 22 février 1638, AT I, 560. 
Si la philosophie première de Descartes se présente sous forme méditative, c'est parce qu'il est nécessaire de pratiquer cet exercice de détachement $^{28}$ et d'attention continuée ${ }^{29}$, pour parvenir à la connaissance claire et distincte des attributs divins ${ }^{30}$. De méditation en méditation, jour après jour, l'entendement progresse dans la connaissance de Dieu : " Je me suis tellement accoutumé ces jours passés à détacher mon esprit des sens, et j’ai si exactement remarqué qu'il y a fort peu de choses que l'on connaisse avec certitude touchant les choses corporelles, qu'il y en a beaucoup plus qui nous sont connues touchant l'esprit humain, et beaucoup plus encore de Dieu même ". Répétés, cet exercice de détachement et cette méditation attentive peuvent conduire au contentement, lequel consiste à placer la considération de l'éternel avant celle du temporel : « (...) maintenant je détournerai sans aucune difficulté ma pensée de la considération des choses sensibles ou imaginables, pour la porter à celles qui, étant dégagées de toute matière, sont purement intelligibles $»^{31}$.

La connaissance de soi en tant que res cogitans est nécessaire à la connaissance conforme de Dieu. Par la méthode du détachement, dont le " premier " fruit est le Cogito et le "second ", l'intellection de la nature divine -, Descartes rompt avec la logique formelle et stérile de l'École. En effet, il existe une différence radicale entre l'épistémologie cartésienne qui pose pour principe le Cogito - c'est-à-dire l'existence certaine d'un être et la scolastique qui part d'une notion commune générale pour connaître indistinctement l'existence de tous les êtres : « (...) la façon dont on réduit les autres propositions à celle-ci : impossibile est idem simul esse et non esse, est superflue et de nul usage ; au lieu que c'est avec une très grande utilité qu'on commence à s'assurer de l'existence de Dieu, et ensuite de celle de toutes les créatures, par la considération de sa propre existence ${ }^{32}$.

Le terme de méditation désigne cet exercice spéculatif de l'entendement où sujet et objet appartiennent à une même nature, la nature intellectuelle en général. Si nous nous accoutumons à nous détourner du sensible, nous pour-

28. "Mais lorsque je relâche quelque chose de mon attention, mon esprit se trouvant obscurci et comme aveuglé par les images des choses sensibles, ne se ressouvient pas facilement de la raison pourquoi l'idée que j'ai d'un être plus parfait que le mien doit nécessairement avoir été mise en moi par un être qui soit en effet plus parfait ", Troisième Méditation, AT IX, 38.

29. «Quoique, pour bien concevoir cette vérité [de l'existence de Dieu], j’aie eu besoin d'une grande application d'esprit... ", Cinquième Méditation, AT IX, 55 ; "Ceux qui considèrent attentivement (attendere) chacune de ses perfections... ", ibid, AT IX, 90, VII, 114.

30. «Et pour ce qui est de Dieu, certes, si mon esprit n'était prévenu d'aucun préjugé, et que ma pensée ne se trouvât point divertie par la présence continuelle des images des choses sensibles, il n'y aurait aucune chose que je connusse plutôt ni plus facilement que lui ", Cinquième Méditation, AT IX, 54-55.

31. Les deux citations renvoient à la Quatrième Méditation, AT IX, 43.

32. A Clerselier, juin ou juillet 1646, AT IV, 445. «J'ai pris l'être ou l'existence de cette pensée pour le premier principe, duquel j'ai déduit très clairement les suivants, à savoir qu'il y a un Dieu... ", Principes, Préface, AT IX, 10. 
rons, en connaissant notre nature, connaître par là même celle de Dieu : car, pour qui sait méditer, l'idée de Dieu (ou d'une nature intellectuelle infinie) est antérieure à la notion de fini ${ }^{33}$. La difficulté à avoir une intuition de la nature divine est liée, non pas seulement à un attachement quasi atavique au sensible, mais au fait d'ériger cet attachement en principe, d'en faire le fondement d'une épistémologie :

(...) ce qui fait qu'il y en a plusieurs qui se persuadent qu'il y a difficulté à le [Dieu] connaître, et même aussi à connaître ce que c'est que leur âme, c'est qu'ils n'élèvent jamais leur esprit au-delà des choses sensibles (...) Ce qui est assez manifeste de ce que même les philosophes tiennent pour maxime, dans les écoles, qu'il n'y a rien dans l'entendement qui n'ait premièrement été dans les sens, où toutefois il est certain que les idées de Dieu et de l'âme n'ont jamais étééc.

Si la théorie de l'innéité des idées et notamment de l'idée de Dieu est une réaction à la maxime scolastique, l'ordre de la méditation demande donc de rompre d'abord avec cette philosophie qui érige en " maxime " l'attachement aux sens ${ }^{35}$. De façon plus générale, une "éducation » ou une "phénoménologie » de l'esprit semble indispensable pour que la connaissance de Dieu et de ses attributs puisse se transformer en une intuition ${ }^{36}$. Les démonstrations cartésiennes apparaîtront alors " plus claires en elles-mêmes qu'aucune des démonstrations des géomètres ; en sorte qu'elles ne me semblent obscures qu'au regard de ceux qui ne savent pas abducere mentem a sensibus ${ }^{37}$. La méditation est une opération de l'âme et, en cela, elle détermine notre pouvoir le plus propre ${ }^{38}$. De plus, elle n'est pas seulement une méthode : elle est l'élément d'une morale. En effet, c'est par elle que se corrige notre propension à croire que le monde est fait pour nous. L'exercice méditatif transforme le "droit illimité sur tous et toutes choses » ${ }^{39}$, "que nos appétits et nos passions nous dictent continuellement », par le pouvoir

33. AT IX, 41.

34. Discours, AT VI, 37. Souligné par nous.

35. « Mais je ne pouvais mieux traiter cette matière, qu'en expliquant amplement la fausseté ou l'incertitude qui se trouve en tous les jugements qui dépendent du sens ou de l'imagination, afin de montrer ensuite quels sont ceux qui ne dépendent que de l'entendement pur, et combien ils sont évidents et certains ", A Mersenne, 27 février 1637, AT I, 350-351.

36. «Mais, outre l'empêchement des préjugés, dont aucun n'est entièrement exempt, bien que ce sont ceux qui ont le plus étudié les mauvaises sciences auxquels ils nuisent le plus, il arrive presque toujours que ceux qui ont l'esprit modéré négligent d'étudier ", Principes, Lettre-Préface, AT IX-B, 12-13.

37. A Mersenne, 27 février 1637, AT I, 350-351. L'existence de Dieu « a été mise en doute par quelques uns, à cause qu'ils ont trop attribué aux perceptions des sens, et que Dieu ne peut être vu ni touché ", Principes, Lettre-Préface, AT IX-B, 10.

38. Car « il n'y a rien qui soit entièrement en notre pouvoir que nos pensées; au moins en prenant le mot de pensée comme je fais, pour toutes les opérations de l'âme, en sorte que non seulement les méditations (...) », A Reneri pour Pollot, avril ou mai 1638, AT II, 37.

39. «Nous nous sommes insensiblement persuadés que le monde n'était fait que pour nous, et que toutes choses nous étaient dues ", ibid. 
réel que nous avons sur nos pensées : "Or nonobstant qu'il soit vrai qu'aucune chose extérieure n'est en notre pouvoir, qu'en tant qu'elle dépend de la direction de notre âme, et que rien n'y est absolument que nos pensées (...) j'ai dit néanmoins qu'il faut s'accoutumer à le croire, et même qu'il est besoin à cet effet d'un long exercice et d'une méditation souvent réitérée ».

\section{La Méditation comme opération intellectuelle propre à la connaissance de Dieu}

La connaissance des attributs divins engage une modalité gnoséologique spécifique qui s'exprime par le terme d'intelligere ${ }^{40}$. L'acte mental auquel renvoie le terme d'intelligere caractérise la connaissance de Dieu qui, parfaitement claire et distincte, ne peut être ni une conception ni une compréhension. En tant qu'idée de l'infini, l'idée de Dieu est la plus claire et la plus distincte $^{41}$. Or, la clarté implique l'attention ${ }^{42}$ et la distinction renvoie à l'acte d'intellection, opposé à celui de compréhension : elle signifie non seulement que nous ne mettons dans l'idée de Dieu que ce qui appartient à Sa nature ${ }^{43}$, mais que nous savons que cette idée n'exhausse pas la nature divine ${ }^{44}$.

Les Principes emploient le terme de respicientes (que le français traduit par faisant réflexion) pour désigner l'acte par lequel nous connaissons les attributs de Dieu ${ }^{45}$. Dans les Réponses aux Septièmes Objections, le terme de réflexion renvoie à celui de consideratio, que Descartes oppose à la simple cogitatio $^{46}$ : la connaissance des attributs est connaissance réflexive et non simple pensée ${ }^{47}$. La consideratio implique l'attention, une contention particulière

40. AT VII, 40 ; 45. "Postquam fatis accurate investigavimus quid sit Deus, clare et distincte intelligimus ad ejus veram \& immutabilem naturam pertinere ut existat ", AT VII, 116. "Per infinitam substantiam, intelligo... ", A Clerselier, 23 avril 1649, AT IX, 356.

41. AT IX, 36-37, maxime clara et distincte, VII, 46.

42. "Claram voco illam, quœe menti attendenti prosens et aperta est ", Principes I, article 45, AT VIII-A, 22.

43. "Distinctam autem illam, quœe, cum clara sit, ab omnibus aliis ita sejuncta est \& procisa, ut nibil plane aliud, quam clarum est, in se contineat ", ibid.

44. «Car il est de la nature de l'infini que ma nature (...) ne le puisse comprendre (non comprehendatur) ; il suffit que j'entende (intelligere) bien cela, et que je juge que toutes les choses que je perçois, percipio, clairement, et dans lesquelles je sais (scio) qu'il y a quelque perfection (...), sont en Dieu », AT IX, 37, VII, 46.

45. "Nous recevons encore cet avantage, en prouvant de cette sorte, per ejus scilicet ideam, l'existence de Dieu, que nous connaissons par même moyen ce qu'il est, autant que le permet la faiblesse de notre nature ? Car, faisant réflexion, respicientes, sur l'idée que nous avons naturellement de lui, nous voyons qu'il est éternel, tout-connaissant, tout-puissant, source de toute bonté et vérité, créateur de toutes choses ", Principes, Première Partie, article 22.

46. "Nec magis etiam similis consideratio sive reflexio requitur, ut substantia cogitans sit posita supra materiam ", AT VII, 559. Souligné par nous.

47. Sur la définition de cogitatio, voir AT IX, 29, exposé géométrique, AT IX, 124, VII, 160, Principes I, article 9. 
de l'esprit ${ }^{48}$; elle définit l'acte mental engagé dans la connaissance des attributs divins ${ }^{49}$. La considération attentive ou réflexion est la modalité par laquelle nous accédons à la connaissance de Dieu. Elle implique un travail de détachement des sens et une persistance dans la perceptio de l'entendement pur, c'est-à-dire la capacité à ne pas divertir la pensée par la " présence continuelle des images des choses sensibles ${ }^{50}$. Jusque dans les Principes, Descartes met donc en ouvre la forme méditative comme méthode de détachement de l'esprit des sens. Une fois adoptée la méthode méditative (abducere mentis a sensibus), la connaissance de Dieu se présente comme aisée : "Je ne vois point en cela de difficulté pour ceux qui ont accoutumé leur esprit à la contemplation de la Divinité, et qui ont pris garde à ses perfections infinies : car encore que nous ne les comprenions pas (...), nous les concevons néanmoins plus clairement et plus distinctement que les choses matérielles ${ }^{51}$.

La facilité avec laquelle les perfections divines peuvent se dévoiler à l'entendement attentif n'est cependant pas à la portée de tous ${ }^{52}$. Les difficultés, qui empêchent la réflexion sur l'idée de Dieu de se déployer dans son évidence, sont des habitudes auxquelles la métaphysique cartésienne va substituer d'autres habitudes : l'entendement accoutumé à distinguer l'essence de l'existence doit désormais envisager que toute essence implique l'existence (possible) et que seule l'essence divine implique l'existence nécessaire. Il convient également de voir en quoi les idées innées ne sont pas des définitions nominales, mais appartiennent, en quelque sorte, au domaine de l'ens reale $^{53}$. La connaissance intuitive n'est pas exclusive d'une grande application de l'esprit, mais elle exige un esprit détaché du commerce des sens et libéré de tout préjugé ${ }^{54}$.

Si Descartes est d'accord avec Thomas d'Aquin pour refuser de faire de l'existence de Dieu une connaissance per se nota ${ }^{55}$; ce n'est cependant pas au nom d'un interdit théologique (l'entendement humain ne peut rien énoncer

48. "Atque, quamvis mibi attenta consideratione opus fuerit ac hoc ipsum percipiendum ", Principes I, article 29, « (...) ad singulas [perfectiones] attendam ", AT VII, 67.

49. «(...) ejus attributorum consideratione ", AT VIII-A, 38.

50. AT IX, 54-55.

51. Principes I, article 19. La méthode méditative de détachement de l'esprit des sens n'est pas exclusive d'un mouvement de "redescente » des objets de l'entendement pur (comme le sont les attributs de Dieu) au monde sensible : "Il suffit d'en prendre une fois une connaissance générale, puis de se souvenir de la conclusion ; autrement elles détournent trop l'esprit de la physique et des choses sensibles, et le rendent impropre à les examiner ", Entretien, AT V, 165, 1. 4-5.

52. "(...) fateor esse difficultatem non parvam ", AT VII, 116, IX, 92.

53. AT IX, 93-94.

54. AT VII, 69, IX, 54-55.

55. «Car on lui demande, savoir, si la connaissance de l'existence de Dieu est si naturelle à l'esprit humain qu'il ne soit pas besoin de la prouver, c'est-à-dire si elle est claire et manifeste à chacun ; ce qu'il nie, et moi avec lui ", AT IX, 91. Souligné par nous. 
de positif sur l'essence de Dieu), mais en raison d'une faiblesse intellective ${ }^{56}$. L'incapacité à concevoir que la réalité objective de l'idée de Dieu requiert une cause et la difficulté à entendre la mineure du syllogisme des Réponses aux Premières Objections témoignent d'une telle faiblesse ${ }^{57}$. De même que les paralogismes de certains mathématiciens peuvent me faire douter de la capacité de l'entendement humain à connaître les choses les plus évidentes ${ }^{58}$; de même l'indigence de la lumière naturelle de certains oblige à donner une démonstration de l'existence de Dieu, laquelle est pourtant de droit manifeste (certissimum videtur) ${ }^{59}$. La conclusion du syllogisme est connue per se nota pour ceux qui fondent la connaissance de Dieu sur une épistémologie d'entendement pur. Par là, il faut entendre une épistémologie détachée du commerce des sens, libérée des préjugés, lesquels consistent non seulement à séparer l'essence de l'existence, à ne pas entendre l'existence de Dieu comme nécessaire mais aussi à penser que la connaissance de Dieu ne peut être que négative, à ne pas percevoir la spécificité de l'idée innée de Dieu, qui nous dispense de tout recours aux choses sensibles pour connaître sa nature.

Tout en reconnaissant l'incompréhensible infinité du Créateur, la méditation donne la connaissance claire de sa nature, connue « avec plus de facilité, qu'il ne s'en trouve en aucune chose créée $"^{60}$. La préparation qui doit conduire l'esprit à se rendre " maître de son attention " ${ }^{61}$ constitue les prémisses nécessaires à toute connaissance de Dieu, y compris à une connaissance intuitive de sa nature : " Même, en s'arrêtant assez longtemps sur cette méditation, on acquiert peu à peu une connaissance (...) intuitive de la nature intellectuelle (...) sans limitation », c'est-à-dire Dieu ; " Je demande qu'ils s'arrêtent longuement à contempler la nature de l'être souverainement parfait (...). Car, de cela seul, et sans aucun raisonnement, ils connaîtront que Dieu existe " ${ }^{62}$. L'application de l'esprit, qui résout le mouvement vers Dieu en une intuition, n'empêche pas la connaissance des attributs divins d'être parfaite (nota \& certa $\left.{ }^{63}\right)$ : «Et quoique, pour bien concevoir cette vérité, $\mathrm{j}$ 'aie eu besoin d'une grande application de l'esprit, toutefois à présent je ne m'en tiens pas seulement aussi assuré que de tout ce qui me semble certain : mais, outre cela, je remarque que la certitude de toutes les choses en

56. «Et toutefois, en faveur de ceux dont la lumière naturelle est si faible, pro iis quorum lumen naturale tam exiguum... ", AT IX, 107, VII, 136.

57. «Il ne reste plus que la mineure, où je confesse que la difficulté n'est pas petite ", premièrement parce que nous avons l'habitude de distinguer l'existence de l'essence et, deuxièmement, parce que nous ne distinguons pas les propriétés qui appartiennent à la vraie et immuable chose de celles qui lui sont attribuées par fiction, AT IX, 91, VII, 116.

58. «Et même, comme je juge quelquefois que les autres se méprennent, même dans les choses qu'ils pensent savoir avec le plus de certitude ", AT IX, 16.

59. AT VII, 69, IX, 55.

60. AT IX, 90.

61. Ibid.,

62. Respectivement A Silhon ( ?), 1637, AT I, 353 ; AT IX, 126.

63. Cinquième Méditation, AT VII, 56. 
dépend si absolument, que sans cette connaissance il est impossible de ne pouvoir jamais rien savoir parfaitement ${ }^{64}$.

Un passage des Réponses aux Secondes Objections est à ce propos révélateur : il tend à accorder à toute preuve (y compris aux deux formulations de la preuve par les effets) une évidence qui devrait nous dispenser, dans la connaissance de Dieu, du détour par le raisonnement :

Or, qu'il y ait en nous quelque idée d'un être souverainement puissant et parfait, et aussi que la réalité objective de cette idée ne se trouve point en nous, ni formellement, ni éminemment, cela deviendra manifeste à ceux qui y penseront sérieusement, et qui voudront avec moi prendre la peine d'y méditer (...) Or de tout cela on conclut très manifestement que Dieu existe. Et toutefois, en faveur de ceux dont la lumière naturelle est si faible qu'ils ne voient pas que c'est une première notion que toute la perfection qui est objectivement dans une idée doit être réellement dans quelqu'une de ces causes, je l'ai encore démontré d'une façon plus aisée à comprendre, en montrant que l'esprit qui a cette idée ne peut exister par soi-même ${ }^{65}$.

\section{La Méditation comme « joie extrême »}

La forme méditative de la connaissance cartésienne des attributs divins signifie que cette connaissance est d'un genre spécifique : "Aussi il n'y a point de spéculation qui puisse plus aider à perfectionner notre entendement, et qui soit plus importante que celle-ci, d'autant que la considération d'un objet qui n'a point de bornes en ses perfections nous comble de satisfaction et d'assurance ${ }^{66}$. Dans cette connaissance, l'entendement se réjouit de la réalité formelle comme de la réalité objective de l'idée. Mais déjà, la conclusion de la Troisième Méditation, sous la notion de marque de l'ouvrier sur son ouvrage, montrait que la connaissance de soi est indissociablement connaissance de Dieu ${ }^{67}$. La méditation de l'idée de

64. Cinquième Méditation, AT IX, 55. «Et ainsi, je reconnais très clairement que la certitude et la vérité de toute science dépend de la seule connaissance du vrai Dieu : en sorte qu'avant que je le connusse, je ne pouvais savoir parfaitement aucune chose ", ibid., AT IX, 56. «( (...) de la connaissance de sa nature, nous passons à l'explication des choses qu'il a créées ", «(...) nous serons assurés, par ceux de ses attributs dont il a voulu que nous ayons quelque connaissance... ", Principes I, articles 24 et 28, AT IX, 35, 37.

65. AT IX, 107. Souligné par nous.

66. "Quia cogitationem nostram magis implent, suntque simpliciores, nec limitationibus ullis obscurantur », Principes I, article 19, AT VIII-A, 12.

67. «Et certes on ne doit pas trouver étrange que Dieu, en me créant, ait mis en moi cette idée pour être comme la marque de l'ouvrier empreinte sur son ouvrage; et il n'est pas nécessaire que cette marque soit quelque chose de différent de ce même ouvrage. Mais de cela seul que Dieu m'a créé, il est fort croyable qu'il m'a en quelque sorte produit à son image et semblance, et que je conçois cette ressemblance (dans laquelle l'idée de Dieu se trouve contenue) par la même faculté par laquelle je me conçois moi-même ", Troisième Méditation, AT IX, 41. 
Dieu $^{68}$ détermine le genre le plus parfait de connaissance car elle se rapporte à la simplicité de Dieu et à l'unité qui contient et embrasse l'ensemble de ses attributs :

(...) encore que nous ne les [perfections infinies de Dieu] comprenions pas, parce que la nature de l'infini est telle que des pensées finies ne le sauraient comprendre, nous les entendons, possumus intelligere, néanmoins plus clairement et plus distinctement que les choses matérielles, à cause qu'étant plus simples, suntque simpliciores, et n'étant point limitées, ce que nous en entendons est beaucoup moins confus ${ }^{69}$.

C'est parce que la capacité de l'entendement à s'élever à l'infini divin est en soi la preuve de l'existence de cet infini que la démonstration trouve son sens dans la contemplation qui lui succède. Aussi, la méditation s'achève-telle nécessairement sur une contemplation. C'est l'infini qui saisit l'entendement et non celui-ci qui parvient à sa compréhension (les termes d'admirer et de contempler sont employés à la fois dans la Troisième Méditation et dans les Réponses aux Premières Objections pour désigner le rapport de l'esprit à l'incompréhensible divin $)^{70}$. Au cœur même de la preuve qui donne la connaissance de l'essence divine ${ }^{71}$, l'entendement découvre les limites de cette connaissance en même temps qu'il reconnaît le Dieu de la foi qu'il faut "admirer et adorer " ${ }^{72}$. L'élévation de l'esprit vers Dieu demande, une fois démontrée son existence, de se soumettre et d'admirer son immense lumière et sa merveilleuse beauté ${ }^{73}$. Dans l'article 19 des Principes, l'entendement humain n'est pas conçu comme une "partie de l'entendement divin », puisque le rapport de créature à Créateur est l'horizon à partir duquel est définie une connaissance des attributs divins. Mais cet article définit une connaissance, où la pensée de l'objet (les perfections divines) est aussi la pensée du

68. La conclusion de la Troisième Méditation part de " Mais, auparavant que j'examine cela plus soigneusement et que je passe à la considération des autres vérités que l'on en peut recueillir, il me semble très à propos de m'arrêter à la contemplation de ce Dieu tout parfait " jusqu'à la fin, AT IX, 41-42. La conclusion de la Cinquième Méditation part de «Et ainsi je reconnais très clairement que la certitude et la vérité de toute science dépend de la seule connaissance du vrai Dieu : en sorte qu'avant que je le connusse, je ne pouvais savoir parfaitement aucune chose. Et à présent que je le connais, j’ai le moyen d'acquérir une science parfaite touchant une infinité de choses, non seulement de celles qui sont en lui, mais aussi de celles qui appartiennent à la nature corporelle », AT IX, 56.

69. Principes I, article 19, AT VIII-A, 12.

70. AT IX, 90.

71. "Parce que cette idée me fait connaître ce que c'est que Dieu », AT IX, 85.

72. AT IX, 41.

73. " At illa veritatis et sapientice pulchritudo, tantum adsit perseverans voluntas fruendi (...) nullus de illa judicat, nullus sine illa judicat bene. Ac per hoc eam manifestum est mentibus nostris, quoe ab ipsa una funt singuloe sapientes et non de ipsa sed per ipsam de ceteris judices, sine dubitatione esse potiorem ", De libero arbitrio, op. cit., II, 14, 38, p. 346. Comparer avec Descartes : "(...) immensi hujus luminis pulchritudinem, quantum caligantis ingenii mei acies ferre poterit, intueri, admirari, adorare ", AT VII, 52. 
sujet : en pensant les perfections de Dieu, c'est la propre perfection de mon entendement que j'éprouve et confirme.

Notre rapport premier à Dieu est l'admiration, c'est-à-dire cet éblouissement de l'esprit ou "surprise de l'âme qui fait qu'elle se porte à considérer avec attention les objets qui lui semblent rares et extraordinaires " ${ }^{74}$. La fin de la Troisième Méditation n'annonçait-elle pas un tel mouvement en déclarant : «(...) il me semble très à propos de m'arrêter quelque temps à la contemplation de ce Dieu tout parfait, de peser tout à loisir ses merveilleux attributs, de considérer (...) l'incomparable beauté de cette immense lumière ${ }^{75}$. S'arrêter à la contemplation de ce Dieu parfait correspond à l'application de notre entendement, que notre volonté détermine à une attention et réflexion particulières ${ }^{76}$.

Cette application de l'entendement fait que, dans la contemplation de Dieu et de ses attributs, la connaissance intuitive ${ }^{77}$ est indissociablement joie : « (...) une semblable méditation (...) nous fait jouir du plus grand contentement que nous soyons capables de ressentir en cette vie ». La réflexion (activité volontaire et intellectuelle de l'âme) peut néanmoins suppléer à l'admiration :

C'est pourquoi, encore qu'il soit bon d'être né avec l'inclination à cette passion, parce que cela nous dispose à l'acquisition des sciences, nous devons toutefois tâcher par après de nous en délivrer le plus qu'il est possible. Car il est aisé de suppléer à son défaut par une réflexion et attention particulière, à laquelle notre volonté peut toujours obliger notre entendement lorsque nous jugeons que la chose qui se présente en vaut la peine ${ }^{78}$.

Le mouvement d'arrêt de l'entendement face au merveilleux des attributs divins constitue une telle réflexion et attention (ou méditation) - exprimées au travers du terme d'intueri. L'objet unique d'une admiration, qui peut s'accompagner d'une connaissance claire et distincte, est donc l'infini divin. Il existe entre, d'une part, l'intelligibilité et l'incompréhensibilité de Dieu et, d'autre part, sa connaissance réflexive et son admiration une structure en chiasme.

La méditation comme suppléant naturel de l'admiration peut s'appliquer à la connaissance de Dieu. Aussi, la contemplation admirative qui conclut la Troisième Méditation trouve-t-elle son prolongement dans le processus réflexif, décrit par l'article 22 des Principes. Pour acquérir une science des attributs divins ${ }^{79}$ et non pas simplement les admirer, il faut que l'entendement soit déterminé par la volonté à une " attention et réflexion

74. Traité des Passions, article 70. Souligné par nous.

75. Troisième Méditation, AT IX, 41.

76. Traité des Passions, article 75.

77. "Intueri », AT VII, 52.

78. Traité des Passions, Seconde Partie, article 76.

79. Cinquième Méditation, AT IX, 56 ; Traité des Passions, Seconde Partie, article 76. 
particulière ${ }^{80}$. L'admiration de la Troisième Méditation, en devenant réflexion sur l'idée de Dieu et connaissance de ses attributs, transforme la contemplation de Dieu en spéculation: "Aussi il n'y a point de spéculation qui puisse plus aider à perfectionner notre entendement, et qui soit plus importante que celle-ci ${ }^{81}$.

Concluons de notre étude de l'article 19 des Principes qu'il existe entre la joie et la connaissance méditative des attributs divins, un lien analytique : connaître Dieu nous procure une satisfaction - sans doute la plus grande dont nous soyons capables en cette vie. La récompense attachée à une telle connaissance ne vient ni ultérieurement ni de l'extérieur : la connaissance de Dieu est à elle-même sa propre récompense.

\section{L'augustinisme de la forme méditative}

À défaut de leur restituer leur horizon historique, ces conclusions contemplatives paraîtraient irréductiblement hétérogènes aux réquisits cartésiens de clarté et de distinction. Or, l'association de preuves rationnelles de l'existence de Dieu à une admiration de la souveraineté divine est une des caractéristiques des démonstrations augustiniennes de l'existence de Dieu. En s'appuyant sur la preuve de l'existence de Dieu présentée dans le De Libero arbitrio $^{82}$, il est possible de conclure que la connaissance de Dieu, l'élévation de l'esprit humain à la connaissance de l'être divin emprunte, chez Descartes, une voie qu'Augustin a dégagée. Cette voie se caractérise à la fois par un refus des effets sensibles comme point de départ d'une telle connaissance, par la présence intime de Dieu en l'homme et par la contemplation de Dieu comme Vérité immuable. Ces exigences épistémologiques de détachement de l'esprit des sens ne renvoient-elles pas à la méthode méditative prônée par Descartes ? La forme méditative de la métaphysique cartésienne, en tant qu'elle est un exercice de détachement des sens, révèlerait ainsi sa provenance augustinienne.

Si Descartes affirme être le premier à appliquer radicalement cette méthode en métaphysique, il reconnaît néanmoins avoir des prédécesseurs :

(...) car, encore qu'il ait été dit par plusieurs que, pour bien entendre les choses immatérielles ou métaphysiques, il faut éloigner son esprit des sens, néanmoins personne, que je sache n'avait encore montré par quel moyen cela se peut faire. Or le vrai et, à mon jugement, l'unique moyen pour cela est contenu dans ma

80. Traité des Passions, Seconde Partie, article 75.

81. Ibid., article 19, AT VIII-A, 12.

82. Notons que si Descartes lit le De Libero arbitrio, il semble ignorer les textes plus tardifs, notamment les Retractationes. Soulignons que dans le De Libero arbitrio, Augustin a pour adversaires les manichéens et non les pélagiens - qui n'existaient pas encore - et que les développements sur le libre-arbitre sont donc différents de ce qu'ils seront ultérieurement. 
seconde Méditation ; mais il est tel que ce n'est pas assez de l'avoir envisagé une fois, il le faut examiner souvent et le considérer longtemps, afin que l'habitude de confondre les choses intellectuelles avec les corporelles, qui s'est enracinée en nous pendant tout le cours de notre vie, puisse être effacée par une habitude contraire de les distinguer, acquise par l'exercice de quelques journées ${ }^{83}$.

Or, dans le De Ordine, Augustin accorde à la connaissance de l'âme par elle-même une semblable fonction :

La cause la plus importante de cette erreur est que l'homme est un inconnu pour lui-même. S'il veut cependant se connaître, il a besoin d'une grande habitude de se retirer des sens (recedendi a sensibus), de recueillir son esprit en lui-même et de le maintenir en lui-même. C'est ce qu'obtiennent seulement ceux qui cautérisent par la solitude ou soignent par des études libérales certaines blessures que causent des opinions et que le cours de la vie quotidienne leur inflige ${ }^{84}$.

Dans le De Libero arbitrio, l'étape du nombre et des règles immuables qui ne dépendent pas de la connaissance sensible sont une étape nécessaire dans la preuve de l'existence de Dieu :

Que les nombres eux-mêmes ne sont pas tirés des sens corporels, tu le verras facilement, si tu penses que tout nombre doit son nom au nombre de fois qu'il possède l'un (...). J'en termine avec les exemples de ce genre. Il suffit que tu voies comme je le vois moi-même et que tu reconnaisses comme certain que ces règles, en somme, ces lumières de vertus sont vraies et immuables, et qu'elles sont chacune ou toutes, à la disposition de la contemplation commune de ceux qui sont capables de les voir, chacun par sa raison et par son esprit (...). Mais, si la vérité était égale à nos esprits, elle serait elle-même muable (...). Or, l'esprit doit d'autant plus saisir par l'intelligence qu'il a pu se rendre plus proche et s'unir à la vérité immuable. C'est pourquoi, si elle n'est ni inférieure ni égale, reste qu'elle est supérieure et meilleure ${ }^{85}$.

De même, la démonstration de la Cinquième Méditation s’appuie-t-elle sur les "natures vraies et immuables », pivot de la preuve de l'existence de Dieu :

Et ce que je trouve ici de plus considérable, est que je trouve en moi une infinité d'idées de certaines choses, qui ne peuvent pas être estimées un pur néant, quoique peut-être elles n'aient aucune existence hors de ma pensée, et qui ne sont pas feintes par moi, bien qu'il soit en ma liberté de les penser ou ne les penser pas; mais elles ont leurs natures vraies et immuables. Comme, par

83. Réponses aux Secondes Objections, AT IX, 104.

84. De Ordine, vol. 4, in Euvres de saint Augustin, Bibliothèque augustinienne, Desclée de Brouwer, 1955, p. 77. Pour une étude lexicale des rapports entre Descartes et Augustin, voir Janowski, Zbigniew, Index augustino-cartésien, Paris, Vrin, 2000.

85. De Libero arbitrio, in Saint Augustin, Euvres, I, Bibliothèque de la Pléiade, Paris, Gallimard, 1998, II, 35, p. 473. 
exemple, lorsque j'imagine un triangle, encore qu'il n'y ait peut-être en aucun lieu du monde hors de ma pensée une telle figure, et qu'il n'y en ait jamais eu, il ne laisse pas néanmoins d'y avoir une certaine nature, ou forme, ou essence (natura, sive essentia, sive forma) déterminée de cette figure, laquelle est immuable éternelle, que je n'ai point inventée, et qui ne dépend en aucune façon de mon esprit (...) ne puis-je pas tirer de ceci un argument et une preuve démonstrative de l'existence de $\mathrm{Dieu}^{86}$ ?

La similitude entre les preuves augustinienne et cartésienne apparaît avec le plus netteté dans l'idée que la possibilité même de ce mouvement de l'esprit vers Dieu, l'évidence avec laquelle il définit Dieu comme l'être " auquel personne n'est supérieur » sont, en l'homme, le signe de l'existence de Dieu - comme si Dieu même était l'agent de la preuve. Le pouvoir de l'esprit humain à accéder à Dieu est ainsi le premier témoignage de l'existence de Dieu : « (...) car c'est lui-même qui a donné à ta raison ce sentiment si pieux et si vrai à son égard ${ }^{87}$. La conclusion de la Troisième Méditation montre que le chemin ou l'exercitatio animi qui a conduit à poser la certitude de l'existence de Dieu est commandé par Dieu même, dont l'idée est la «marque de l'ouvrier empreinte sur son ouvrage ». C'est pourquoi Descartes affirme : «(...) je n'ai jamais traité de l'infini que pour me soumettre à lui, et non point pour déterminer ce qu'il est, ou [ce] qu'il n'est pas ${ }^{88}$. Que le pouvoir de l'esprit à s'élever à la connaissance de Dieu soit le premier témoignage de l'existence de Dieu, cela est clairement énoncé par Augustin ${ }^{89}$ et repris avec force par Descartes ${ }^{90}$. Mais ce qui confirme la parenté entre la métaphysique cartésienne et la pensée augustinienne est principalement

86. AT IX, 51, VII, 64. Voir aussi le Discours : «Pour ce qui est des pensées que j’avais de plusieurs autres choses hors de moi (...) ne remarquant rien en elles qui me semblât les rendre supérieures à moi, je pouvais croire que, si elles étaient vraies, c'étaient des dépendances de ma nature (...). Mais ce ne pouvait être le même de l'idée d'un être plus parfait que le mien. ", AT VI, 34. Soulignons que la thématique générale de cette preuve du Discours ainsi que seconde formulation de la preuve par les effets de la Troisième Méditation est comparable à celle qui anime la démonstration du De libero arbitrio, à savoir la preuve de l'existence de quelque chose de plus parfait que moi voire d'absolument parfait, tel que rien de plus parfait ne puisse être pensé : " Je ne suis pas prêt, si je peux trouver quelque chose de meilleur que ce qu'il y a de meilleur dans ma nature, à dire que c'est Dieu. Car il ne me plaît pas d'appeler Dieu ce à quoi ma raison est inférieure, mais seulement ce à quoi rien n'est supérieur. — Absolument, car c'est lui-même qui a donné à ta raison de sentir si pieusement et si véritablement à son sujet $(\ldots)$ Je confesserai pleinement que Dieu est celui dont il est établi qu'il n'y a rien de supérieur ", op. cit., II, 17. Comparer avec cette expression de Descartes : « Et je demande, de qui aurais-je mon existence ? Peut-être de moi-même, ou de mes parents, ou bien de quelques causes moins parfaites que Dieu ; car on ne se peut rien imaginer de plus parfait, ni même d'égal à lui. ", AT IX, 38. Souligné par nous. C'est donc dès la Troisième Méditation que se marque le caractère augustino-anselmien de la preuve cartésienne de l'existence de Dieu.

87. De Libero arbitrio, in Saint Augustin, CEuvres, I, II, 14, p. 455.

88. A Mersenne, 28 janvier 1641, AT III, 293.

89. De Libero arbitrio, in Saint Augustin, I, I, II, 34, p. 473 ; II, 14, p. 455.

90. Voir par exemple, A Regius, 24 mai 1640, AT III, 64. 
l'usage commun de la notion d'imago Dei: l'esprit, en tant qu'il a l'idée de Dieu, est à l'image de Dieu. Bien plus qu'une idée, c'est donc un mode de penser qui conduit l'homme à la connaissance de Dieu. C'est sans doute parce qu'elles mettent en œuvre une telle modalité de l'esprit que les preuves cartésiennes sont les meilleures : «L'importance est en ceci que, puisque je soutiens la cause de Dieu, on ne saurait rejeter mes raisons, si ce n'est qu'on y montre du paralogisme, ce que je crois être impossible, ni les mépriser, si ce n'est qu'on en donne de meilleures, à quoi je pense qu'on aura assez de peine ${ }^{91}$.

\section{Augustin et L'ordo meditandi cartésien}

La parenté entre la preuve cartésienne et la démonstration augustinienne ne se limite pas à une méthode - celle qui consiste à détacher l'esprit des sens — mais elle s'étend à la structure même et à l'enchaînement logique des méditations. De fait, le De Libero arbitrio ne consiste pas seulement à présenter une preuve de l'existence de Dieu systématiquement développée ${ }^{92}$, mais à articuler ensemble les questions de l'existence et de la justification de Dieu. L'itinéraire augustinien est tout à la fois théodicée : "Explique-moi pourquoi Dieu a donné à l'homme le libre-arbitre de la volonté ; car, assurément, s'il ne l'avait pas reçu, il ne pourrait pas pécher (...). Je ne m'y oppose nullement. Mais je pose cette autre question : comment sais-tu que nous existons par lui ? » ${ }^{93}$ et élévation de l'esprit à Dieu : « (...) cherchons donc, si tu le veux bien, dans cet ordre : d'abord, comment se fait l'évidence sur l'existence de Dieu ? ensuite, tout ce qui est bon, en quelque mesure qu'il le soit, vient-il de Dieu ? enfin, faut-il compter parmi ces biens la volonté libre ? Cela découvert, il apparaîtra suffisamment, je pense, si cette volonté a été donnée à l'homme à bon droit ${ }^{94}$. Or, c'est le même ordre qui commande les Méditations : la théodicée de la Quatrième Méditation trouve donc légitimement sa place au sein des preuves de l'existence de Dieu ; car si certains font argument de l'existence du mal pour en inférer la non-existence de $\mathrm{Dieu}^{95}$, il convient de démontrer ensemble que Dieu existe et qu'il n'est pas responsable de l'erreur.

91. A Mersenne, 11 novembre 1640, AT III, 240.

92. Nous ne pouvons partager la thèse de F. Van Steenberghen selon laquelle la preuve augustinienne de l'existence de Dieu serait une "tentative inefficace ", in Dieu caché. Comment savons-nous que Dieu existe?, Louvain-Paris, 1966, p. 69-74.

93. De Libero arbitrio, in Saint Augustin, Euvres, I, II, 1, 2-3.

94. De Libero arbitrio, in Saint Augustin, Euvres, I, III, 7. Thomas d'Aquin commente la preuve augustinienne dans la Somme théologique, Ia., qu. 2, a. 3, sol. 1, 2.

95. "Il y aura peut-être ici des personnes qui aimeront mieux nier l'existence d'un Dieu si puissant, que de croire que toutes les autres choses sont incertaines. ", Première Méditation, AT IX, 16. 
Dès la Première Méditation, la métaphysique cartésienne est confrontée à la nécessité d'une théodicée ; en effet, certaines personnes (nonnulli) préfèrent nier l'existence d'un Dieu plutôt que d'admettre la condamnation de l'homme à cette sorte de mal épistémologique qu'est l'erreur ${ }^{96}$. Par conséquent, la Quatrième Méditation, qui résout de façon définitive la question de l'origine de l'erreur et réaffirme l'existence d'un Dieu vérace, ne rompt pas la continuité qui relie la preuve de la Troisième Méditation à celle de la Cinquième Méditation. C'est l'ordre même de méditer qui impose de répondre conjointement aux questions de l'existence de Dieu et de la capacité de l'homme à parvenir à la science.

La Quatrième Méditation présente en quelque sorte une troisième preuve par les effets en tant qu'elle part de la liberté de la volonté pour en conclure, une fois encore, à la nécessaire existence de Dieu. En effet, bien loin de conduire à nier l'existence de Dieu, la considération de notre libre vouloir nous rend évidente notre responsabilité dans l'erreur et, par contraste, la perfection de Dieu : si le Créateur ne m'a pas produit infaillible, il a néanmoins laissé en mon pouvoir la faculté de "ne jamais donner mon jugement sur les choses dont la vérité ne m'est pas clairement connue ${ }^{97}$. La découverte de ce pouvoir ou «perfection » de l'homme témoigne de l'existence de Dieu et de sa grandeur ${ }^{98}$.

Cette théodicée fait voler en éclats l'alternative posée dans la Première Méditation, qui enfermait le narrateur cartésien dans les limites étroites d'une logique binaire (ou Dieu existe et il est responsable de l'erreur ou alors Dieu n'existe pas et l'homme est condamné à l'erreur). Non seulement il est démontré que Dieu n'est pas à l'origine de l'erreur mais il est clairement affirmé que l'effet de sa puissance consiste à m'avoir donné le moyen d'éviter l'erreur et non à m'avoir rendu aveugle à la vérité. L'insuffisance de la connaissance de Dieu, dans la Première Méditation, conduisait à nous contenter de la double affirmation de la toute-puissance divine et de notre impuissance - notre entendement étant le jouet de ce Dieu dont certains préféraient nier l'existence. La solution de la Quatrième Méditation consiste à poser à la fois la toute-puissance du Créateur et la perfection ou capacité de la créature ${ }^{99}$.

La connaissance de la véracité divine, prolongeant la connaissance de Dieu inaugurée avec les preuves de la Troisième Méditation, a permis de parvenir à cette solution. C'est pourquoi la théodicée de la Quatrième Méditation se conclut, comme la preuve de la Méditation précédente, sur la considération de la perfection divine - laquelle exclut que Dieu soit trom-

96. AT IX, 16.

97. AT IX, 49.

98. «Dieu, dis-je, qui, étant souverainement parfait (summe perfectum), ne peut être cause d'aucune erreur », AT IX, 50, VII, 62.

99. AT IX, 49. 
peur ${ }^{100}$. Contrairement à G. Rodis-Lewis ${ }^{101}$, qui soutient l'idée de l'hétérogénéité de la Quatrième Méditation par rapport à l'unité que forment les Troisième et Cinquième Méditations, nous soulignons donc le lien métaphysique qui relie la théodicée de la Quatrième Méditation au système des preuves, relevant par là même l'ordre augustinien des Méditations.

Pour conclure, soulignons l'analogie entre la conclusion des preuves par les effets - qui unit dans une même méditation, la connaissance de Dieu comme fondement de la science ${ }^{102}$ et la contemplation du Dieu incompréhensible de la foi ${ }^{103}$ - et la célébration de la Vérité qui clôt la preuve augustinienne ${ }^{104}$. Chez Descartes, c'est parce que la capacité de l'entendement à s'élever à l'infini divin est en soi la preuve de l'existence de cet infini que la démonstration trouve son sens dans la contemplation qui lui succède. C'est l'infini qui saisit l'entendement et non celui-ci qui parvient à sa compréhension (les termes d'admirer et de contempler sont employés à la fois dans la Troisième Méditation et dans les Réponses aux Premières Objections pour désigner le rapport de l'esprit à l'incompréhensible divin $)^{105}$. Au cœur même de la preuve qui donne la connaissance de l'essence divine ${ }^{106}$, l'entendement découvre les limites de cette connaissance en même temps qu'il reconnaît le Dieu de la foi qu'il faut " admirer et adorer ${ }^{107}$. Chez Descartes comme chez Augustin, l'élévation de l'esprit

100. « Ce même Dieu, dis-je, duquel l'idée est en moi, c'est-à-dire qui possède toutes ces hautes perfections (...) D'où il est assez évident qu'il ne peut être trompeur », AT IX, 41 / "Dieu, dis-je, qui, étant souverainement parfait, ne peut être cause d'aucune erreur », AT IX, 49.

101. Rodis-Lewis, Geneviève, " On the Complementary of Meditations III and V: From the 'general rule' of evidence to 'certain science' ", dans Rorty, Amely, dir., Essays on Descartes' Meditations, University of California Press, 1986, p. 271-295.

102. « (...) il est assez évident qu'il ne peut être trompeur, puisque la lumière naturelle nous enseigne que la tromperie dépend nécessairement de quelque défaut ", AT IX, 41.

103. «Car, comme la foi nous apprend que la souveraine félicité de l'autre vie ne consiste que dans cette contemplation de la Majesté divine, ainsi expérimentons-nous dès maintenant qu'une semblable méditation (...) nous fait jouir du plus grand contentement ", AT IX, 42.

104. " En effet, Dieu existe ; il existe vraiment et suprêmement (vere summeque est) ; et ceci, à mon sens, nous ne le tenons plus seulement pour indubitable par la foi, mais nous l'atteignons aussi par une forme de connaissance certaine, bien qu'elle soit encore très ténue ", De Libero arbitrio, in Saint Augustin, CEuvres, I, II, 15, 39.

105. AT IX, 90.

106. « Parce que cette idée me fait connaître ce que c'est que Dieu », AT IX, 85.

107. AT IX, 41. 
vers Dieu demande, une fois démontrée son existence, de se soumettre et d'admirer ( « (...) cum isti subdimur veritati » $\left.{ }^{108}\right)$ son immense lumière et sa merveilleuse beauté ${ }^{109}$.

108. Ibid., II, 13, 37, p. 342.

109. "(...) sed omnibus incommutabilia vera cernentibus tanquam miris modis secretum et publicum lumen praesto esse ac se praebere communiter ", ibid., II, 12, 34, pp. 334336 ; « At illa veritatis et sapientice pulchritudo, tantum adsit perseverans voluntas fruendi (...) nullus de illa judicat, nullus sine illa judicat bene. Ac per hoc eam manifestum est mentibus nostris, quoe ab ipsa una funt singuloe sapientes et non de ipsa sed per ipsam de ceteris judices, sine dubitatione esse potiorem ", ibid., II, 14, 38, p. 346. Descartes affirme pour sa part : «(...) immensi hujus luminis pulchritudinem, quantum caligantis ingenii mei acies ferre poterit, intueri, admirari, adorare ", AT VII, 52. Cet augustinisme qui anime la conclusion de la Troisième Méditation trouve un écho fidèle dans le Traité de l'existence de Dieu de Fénelon: «(...) votre infini m'étonne et m'accable ; c'est ma consolation : je suis ravi que vous soyez si grand que je ne puisse vous voir tout entier (...) mon esprit succombe sous tant de majesté ; heureux de baisser les yeux, ne pouvant soutenir par mes regards l'éclat de votre gloire ", in Euvres, Bibliothèque de la Pléiade, Paris, Gallimard, 1997, p. 615. Comme Descartes, Fénelon emprunte à Augustin ces louanges, qui rapportent à la souveraineté de Dieu, les preuves dont l'évidence ne doit pas faire oublier la faiblesse de la créature face à l'infinie perfection du Créateur. L'indifférenciation entre le Dieu de la foi et le Dieu des preuves oblige à préserver, au sein même de la rigueur démonstrative de ces dernières, la transcendance de leur objet. 
